\title{
Effects of Cyclic Stretching Exercise on Long-Lasting Hyperalgesia, Joint Contracture, and Muscle Injury Following Cast Immobilization in Rats
}

\author{
Kazuhiro HAYASHI ${ }^{1,2} *$, Saori FUKUYASU-MATSUO ${ }^{3 *}$, Takayuki INOUE ${ }^{4}$, Mitsuhiro \\ FUJIWARA $^{5,6}$, Yuji ASAI ${ }^{7}$, Masahiro IWATA ${ }^{6,7}$, Shigeyuki SUZUKI ${ }^{8}$ \\ * These authors contributed equally to this work.
}

${ }^{1}$ Multidisciplinary Pain Center, Aichi Medical University, Nagakute, Japan, ${ }^{2}$ Department of Rehabilitation, Aichi Medical University Hospital, Nagakute, Japan, ${ }^{3}$ Division of Rehabilitation, Gifu University Hospital, Gifu, Japan, ${ }^{4}$ Department of Rehabilitation, Nagoya University Hospital, Nagoya, Japan, ${ }^{5}$ Department of Rehabilitation, Kamiiida Rehabilitation Hospital, Nagoya, Japan, ${ }^{6}$ Department of Physical and Occupational Therapy, Nagoya University Graduate School of Medicine, Nagoya, Japan, ${ }^{7}$ Department of Rehabilitation, Faculty of Health Sciences, Nihon Fukushi University, Handa, Japan, ${ }^{8}$ Department of Health and Sports Sciences, School of Health Sciences, Asahi University, Mizuho, Japan

Received January 20, 2020

Accepted June 9, 2020

Epub Ahead of Print September 9, 2020

\begin{abstract}
Summary
The effects of exercise on mechanical hyperalgesia, joint contracture, and muscle injury resulting from immobilization are not completely understood. This study aimed to investigate the effects of cyclic stretching on these parameters in a rat model of chronic post-cast pain (CPCP). Seventeen 8-week-old Wistar rats were randomly assigned to (1) control group, (2) immobilization (CPCP) group, or (3) immobilization and stretching exercise $(\mathrm{CPCP}+\mathrm{STR})$ group. In the $\mathrm{CPCP}$ and $\mathrm{CPCP}+\mathrm{STR}$ groups, both hindlimbs of each rat were immobilized in full plantar flexion with a plaster cast for a 4-week period. In the CPCP+STR group, cyclic stretching exercise was performed 6 days/week for 2 weeks, beginning immediately after cast removal prior to reloading. Although mechanical hyperalgesia in the plantar skin and calf muscle, ankle joint contracture, and gastrocnemius muscle injury were observed in both immobilized groups, these changes were significantly less severe in the CPCP+STR group than in the CPCP group. These results clearly demonstrate the beneficial effect of cyclic stretching exercises on widespread mechanical hyperalgesia, joint contracture, and muscle injury in a rat model of CPCP.
\end{abstract}

\section{Key words}

Stretching exercise - Hyperalgesia - Muscle damage • Immobilization

\section{Corresponding author}

M. Iwata, Department of Rehabilitation, Faculty of Health Sciences, Nihon Fukushi University, 26-2 Higashihaemi-cho, Handa, Aichi 475-0012, Japan. Fax: +81-569-20-0127. E-mail: iwata-m@n-fukushi.ac.jp

\section{Introduction}

Chronic periods of reduced physical activity can occur following traumatic injury, with prolonged immobilization, and as a part of aging. The primary effects of muscle disuse in such situations include progressive skeletal muscle atrophy (Honda et al. 2015), loss of muscle extensibility (Honda et al. 2018), and joint contracture (Inoue et al. 2007, Morimoto et al. 2013). Studies have confirmed that 4 weeks of hindlimb cast immobilization causes disuse muscle atrophy in rats (Okita et al. 2009), with decreased capillary-to-myofiber ratios in the hindlimb muscles after 2 weeks (Kataoka 
et al. 2014) and 4 weeks (Matsumoto et al. 2014) of immobilization. Other studies have shown that cast immobilization induces muscle fibrosis, which contributes to limb contracture (Honda et al. 2015, Maezawa et al. 2017, Yoshimura et al. 2017). A 4-week period of hindlimb cast immobilization was shown to increase the vulnerability of rats to muscle damage at reloading because of alterations in mobility and movement (Inoue et al. 2009).

In addition to physical and functional changes, recent studies in healthy human subjects and animal models have found that prolonged immobilization induces pain hypersensitivity (Terkelsen et al. 2008, Nakano et al. 2012, Ohmichi et al. 2012, Morimoto et al. 2013, Sekino et al. 2014, Hamaue et al. 2015, Nakagawa et al. 2018) and may contribute to the development of complex regional pain syndrome (Allen et al. 1999). A study of healthy rats with 2 -week cast immobilization of one hindlimb found long-lasting skin and muscle hyperalgesia in the immobilized and contralateral limbs (chronic post-cast pain; CPCP) (Ohmichi et al. 2012).

Immobilization-induced hyperalgesia and joint contracture affect the recovery of muscle functionality after immobilization, limit activities of daily living, and increase healthcare costs. Various therapeutic strategies for reducing $\mathrm{CPCP}$ and joint contracture, including treadmill exercises (Morimoto et al. 2013), vibration exercises (Hamaue et al. 2015), and static stretching (Morimoto et al. 2013), have been evaluated in animal models. However, the effects of stretching exercise on postimmobilization pain and joint contracture remain unclear. Some studies have found that stretching reduces joint contracture (Kaneguchi et al. 2019), whereas others have not found a clinically relevant effect (Harvey et al. 2017). Continuous passive motion on a stretching machine was shown to decrease markers of inflammation and mitigate hyperalgesia in a rat model of arthritis (Nakabayashi et al. 2016). Similarly, stretching exercises reduced inflammation and improved pain in rats with subcutaneous inflammation induced by carrageenan (Corey et al. 2012). One recent animal study reported that static stretching decreased pain and increased joint range of motion (ROM) in a rat model of CPCP (Morimoto et al. 2013). However, to our knowledge, no studies have evaluated the effect of cyclic stretching initiated immediately after cast removal on post-immobilization muscle pain in a rat model of CPCP. The hypothesis of this study was that cyclic stretching exercises initiated immediately after cast removal would decrease long-lasting post-immobilization mechanical hyperalgesia in rats. We also evaluated the effect of cyclic stretching on post-immobilization joint contracture and muscle damage.

\section{Methods}

\section{Animals}

All experiments were approved by the Ethics Committee for Animal Experimentation at the Nagoya University School of Health Science. This study was performed in compliance with the ethical guidelines of the International Association for the Study of Pain and the European Guidelines on Laboratory Animal Care.

Seventeen 8-week-old male Wistar rats were purchased from Japan SLC (Hamamatsu, Japan) and housed under a 12-h light/dark cycle with free access to food and water. The rats were randomly divided into the following three groups: CPCP without cyclic stretching exercises (CPCP, $n=6), \mathrm{CPCP}$ with cyclic stretching exercises $(\mathrm{CPCP}+\mathrm{STR}, \mathrm{n}=6)$, and age-matched naïve controls (CON, n=5; Fig. 1A).

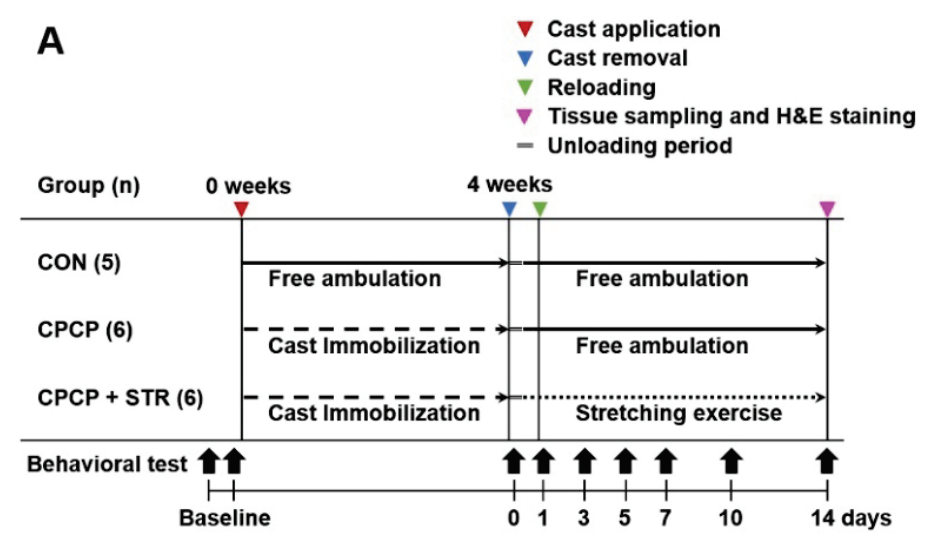

Fig. 1A. Schematic diagram and photos of experimental protocol and representative photomicrographs of muscle tissue (hematoxylin-eosin staining). Treatment groups and treatment schedule. Rats were divided into three groups: age-matched naïve controls (CON, $n=5)$, chronic post-cast pain (CPCP) without cyclic stretching exercise (CPCP, $n=6)$, and CPCP with cyclic stretching exercise $(C P C P+S T R, n=6)$. 


\section{Immobilization and reloading}

CPCP was generated through 4 weeks of hindlimb cast immobilization (Nakagawa et al. 2018). Rats in the CPCP and CPCP+STR groups were anesthetized with intraperitoneal pentobarbital sodium $(40 \mathrm{mg} / \mathrm{kg})$. The bilateral hindlimbs were encased for 4 weeks in plaster casts (Alcare, Tokyo, Japan) in full plantar flexion from just above the knee to the distal foot. Casts were replaced every 2 to 3 days to prevent loosening and hindpaw edema. When the immobilized rats were anesthetized, the age-matched controls (CON group) were also anesthetized to avoid possible confounding. Pentobarbital sodium was the only medication administered during the study period. After the 4-week immobilization period, casts were removed and animals were allowed to ambulate freely in their cages.

\section{Stretching exercises}

Stretching exercises were modified from Inoue et al. (2009). Rats in the CPCP+STR group were anesthetized as above and the bilateral gastrocnemius muscles were stretched with a custom-built apparatus (Fig. 1B). The hindlimb was stabilized with hip and knee extended by taping the foot to the platform, which was connected to a movable board attached to a shaft. The amplitude and frequency of cyclical stretches were controlled with a stepping motor. Stretching exercises were performed at a frequency of once every $4 \mathrm{~s}$ with a range of $40^{\circ}$ from maximum dorsiflexion, as measured with a goniometer. The cyclical stretching was performed for $30 \mathrm{~min} /$ day, 6 days/week, beginning immediately after cast removal (prior to reloading) and continuing for 2 weeks (12 sessions total).
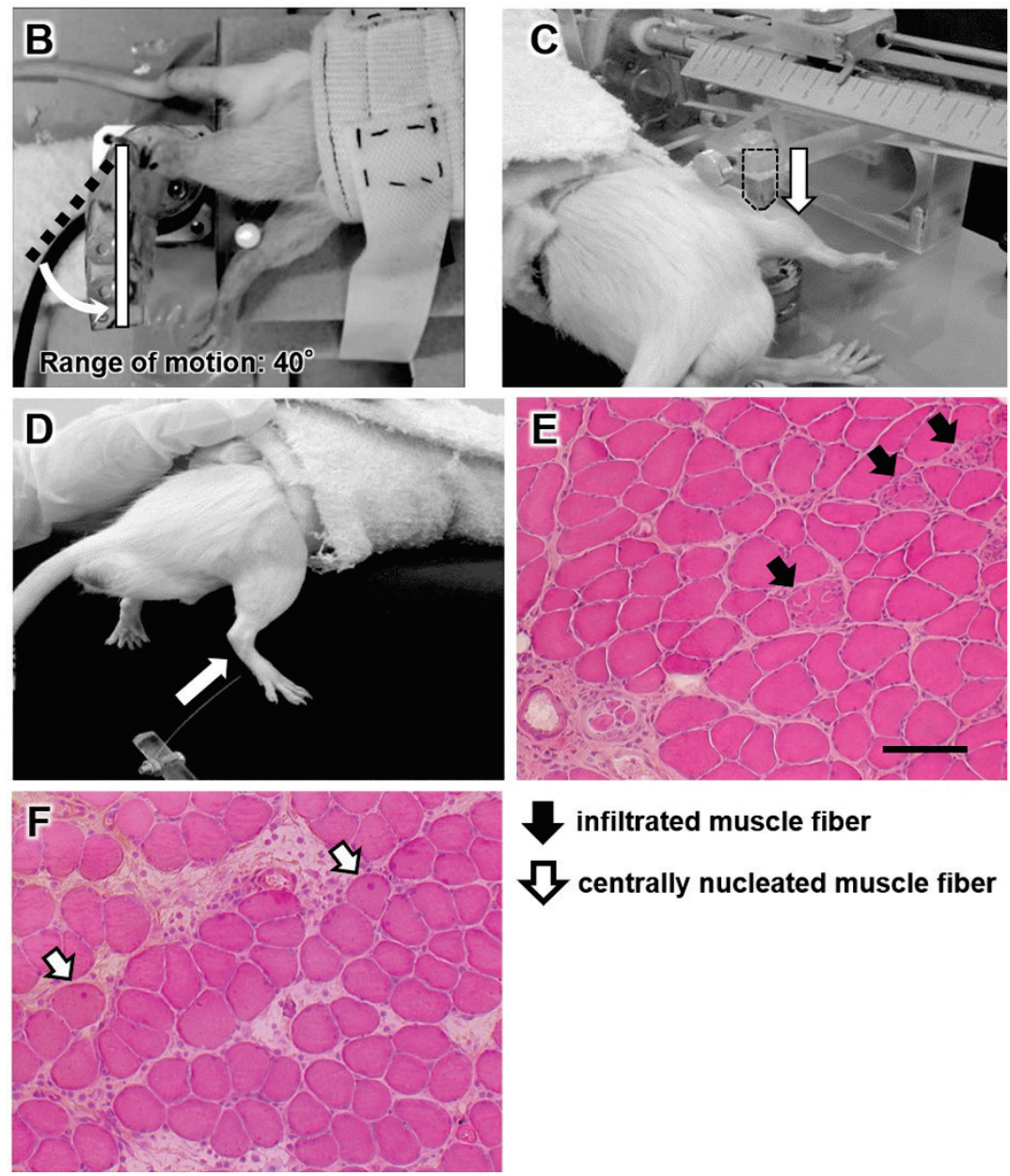

Fig. 1B-F. Schematic diagram and photos of experimental protocol and representative photomicrographs of muscle tissue (hematoxylin-eosin staining). (B) Photograph showing application of stretching exercise. Stretching was performed cyclically in the direction of plantar and dorsiflexion (in the range of $40^{\circ}$ from maximum dorsiflexion) using a stretch apparatus at a frequency of once every $4 \mathrm{~s}$ for $30 \mathrm{~min} /$ day, 6 days/week. (C) Mechanical sensitivity of the gastrocnemius muscle was evaluated with a Randall-Selitto apparatus. (D) Mechanical sensitivity of the glabrous skin of the hindpaw was evaluated with von Frey filaments. (E, F) Representative photomicro-graphs of infiltrated muscle fiber (E) and centrally nucleated muscle fiber (F). Black and white arrows indicate infiltrated fibers and centrally nucleated fibers, respectively. Scale bar, $100 \mu \mathrm{m}$.

\section{Behavior tests}

Behavior tests to assess mechanical sensitivity in the calf muscle and hindpaw skin were performed before cast immobilization (baseline), prior to reloading immediately after cast removal (Day 0), and on Days 1, 3, $5,7,10$, and 14 after cast removal. The tests were 
performed prior to stretching on each testing day. During these tests, rats were wrapped individually in a cloth restrainer because ankle joint contracture prevented those in the immobilized groups from walking on their hindlimbs. As shown in Figures $1 \mathrm{C}$ and $\mathrm{D}$, the restrainer allowed the animal to dangle safely with the legs positioned to be free and under no loading, as described by Nakano et al. (2012).

A Randall-Selitto analgesiometer (Ugo Basile, Comerio, Italy) equipped with a probe with a 2.6-mm tip diameter was used to measure the withdrawal threshold of the right gastrocnemius muscle (Fig. 1C). Use of a large-diameter probe enabled measurement of the withdrawal threshold of deep tissue (Nasu et al. 2010). The nociceptive threshold was defined as the force that induced a withdrawal response to an increasing pressure stimulus from 0 to $250 \mathrm{~g}$. Measurements were repeated seven times at 2- to 3-min intervals; the mean value in each session was taken as the withdrawal threshold.

The glabrous skin of the right hindpaw was probed six times with 2- and 7-g von Frey filaments (VFFs; North Coast Medical, Morgan Hill, CA, USA) at 10-s intervals (Fig. 1D). Lifting or pulling back the paw was counted as a paw withdrawal response. The 2- and 7-g filaments were used to ascertain mechanical allodynia and mechanical hyperalgesia, respectively (Peleshok and Ribeiro-da-Silva 2011). This procedure was performed prior to the Randall-Selitto test on each testing day.

\section{Joint contracture}

Dorsiflexion ROM of the bilateral ankle joints was measured with a goniometer (Inoue et al. 2007). Following the pain behavior tests, the rat was anesthetized and laid on its side with the knee flexed to $90^{\circ}$. The ankle was passively dorsiflexed maximally and the angle formed by the intersection of the line connecting the fifth metatarsal with the malleolus lateralis and that connecting the malleolus lateralis with the center of the knee joint was measured $\left(0^{\circ}-180^{\circ}\right)$.

\section{Histological analysis}

At the end of the experiment, the right gastrocnemius muscle of each animal was excised under anesthesia with intraperitoneal pentobarbital sodium $(50 \mathrm{mg} / \mathrm{kg})$. The muscles were embedded in an optimal cutting temperature compound (TissueTek ${ }^{\circledR}$; Sakura Finetek, Tokyo, Japan), quickly frozen by immersion in isopentane precooled in liquid nitrogen, and processed for sectioning on a cryostat (CM1510-11; Leica, Wetzlar,
Germany). Serial transverse sections $(7 \mu \mathrm{m})$ were cut from the muscle mid-belly and stained with hematoxylineosin to assess muscle injury. Digital images of the stained sections were acquired with an optical microscope (BZ-9000; Keyence, Osaka, Japan) at $\times 400$ magnification (Fig. 1E, F). Five image files were selected with a random number table. Injured muscle fibers were defined as those displaying infiltration by more than two nucleated inflammatory cells (Fig. 1E) (Koh et al. 2003). Central nuclei were defined as those located more than one nuclear diameter from the fiber border; myofibers with central nuclei were termed centrally nucleated fibers (Fig. 1F) (Zschüntzsch et al. 2016). A total of 10,000 muscle fibers contained in five images (image area, $1.5 \times 1.2 \mathrm{~mm}$ ) were analyzed with Image $\mathrm{J}$ software (National Institutes of Health, Bethesda, MD, USA). The number of infiltrated muscle fibers and the number of centrally nucleated fibers per 10,000 fibers were used as indices of muscle injury.

\section{Statistical analysis}

Sigma Plot 13 (Systat Software, San Jose, CA, USA) was used for analyses. Because some dependent variables were not normally distributed according to Shapiro-Wilk testing, non-parametric tests were applied to all variables. The Friedman test was applied to compare differences in outcome measures between timepoints within each group. When a significant difference was found, a Dunnett's post hoc test was performed to identify a significant difference from the baseline value. Differences between groups were analyzed with the Kruskal-Wallis test followed by a Dunn-Bonferroni post hoc test for all pairwise multiple comparisons. $P<0.05$ were considered significant. Graphs plot mean \pm standard error of the mean (SEM), unless noted otherwise.

\section{Results}

\section{Withdrawal thresholds of gastrocnemius muscle}

Withdrawal thresholds immediately after cast removal in the $\mathrm{CPCP}$ and $\mathrm{CPCP}+\mathrm{STR}$ groups were more than $20 \%$ lower than baseline values (from 216 to $165 \mathrm{~g}$ in CPCP group and from 217 to $158 \mathrm{~g}$ in the CPCP+STR group). These threshold values were significantly lower than that of the CON group $(P=0.035$ vs. CPCP and $P=0.013$ vs. $\mathrm{CPCP}+\mathrm{STR}$; Fig. 2). The threshold reduction in the CPCP group was maintained over the 14-day study period and this threshold was always significantly lower 
than that of the CON group $(P=0.002$ on Days 1,3 , and $10 ; P<0.001$ on Day 5; $P=0.004$ on Days 7 and 14). Conversely, the threshold reduction observed in the $\mathrm{CPCP}+\mathrm{STR}$ group gradually recovered. By Day 1 after cast removal, there was no significant difference in threshold level between the $\mathrm{CPCP}+\mathrm{STR}$ and $\mathrm{CON}$ groups. The threshold value of the $\mathrm{CPCP}+\mathrm{STR}$ group was significantly higher than that of the CPCP group at 14 days after cast removal $(P=0.036)$.

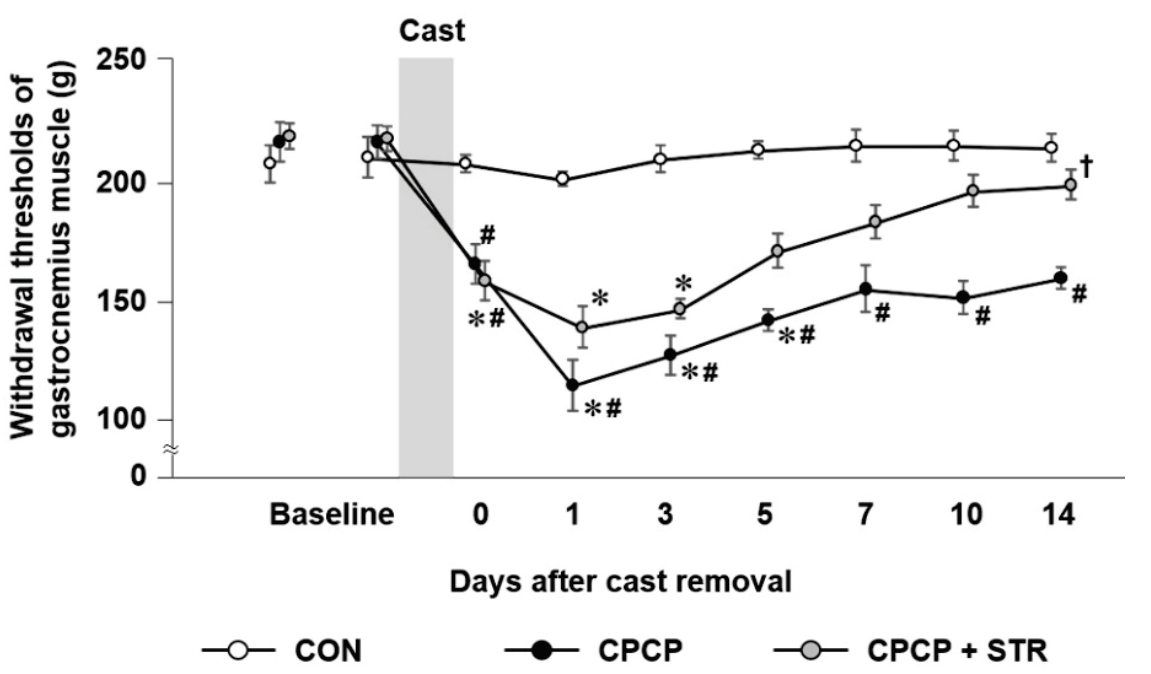

Fig. 2. Time course of changes in withdrawal thresholds of gastrocnemius muscle. Horizontal axis indicates measurement time points. Data are presented as mean \pm SEM ( $n=5$ or 6$)$. $* P<0.05$ relative to associated baseline values; $\# P<0.05$ relative to CON group; ${ }^{+} P<0.05$ relative to $\mathrm{CPCP}$ group.

Paw-withdrawal responses

The number of paw-withdrawal responses elicited with 2-g VFFs is presented in Figure 3A. The number of responses after cast removal did not significantly differ from the number at baseline in any group at any point during the experimental period. However, the number of responses in the CPCP group was significantly higher than that in the CON group on Day 5 after cast removal $(P<0.044)$.

The number of paw-withdrawal responses elicited with a 7-g VFF is presented in Figure 3B. The number of responses in the CPCP group was significantly higher on Days $5(P<0.001)$ and $7(P=0.030)$ after cast removal compared with the number at baseline and was higher than the number in the $\mathrm{CON}$ group on Days 1 $(P=0.008), 5(P=0.001), 7(P=0.002), 10(P=0.002)$, and $14(P=0.006)$ after cast removal. The number of responses in the $\mathrm{CPCP}+\mathrm{STR}$ group was slightly but significantly increased on Day 5 after cast removal compared with baseline $(P=0.018)$; however, this value was not significantly different than that in the CON group.

\section{Range of motion of ankle dorsiflexion}

The ROM of bilateral ankle dorsiflexion is presented in Figures 4A and B. The ROM in both hindlimbs immediately after cast removal was significantly lower than at baseline in the CPCP and CPCP+STR groups

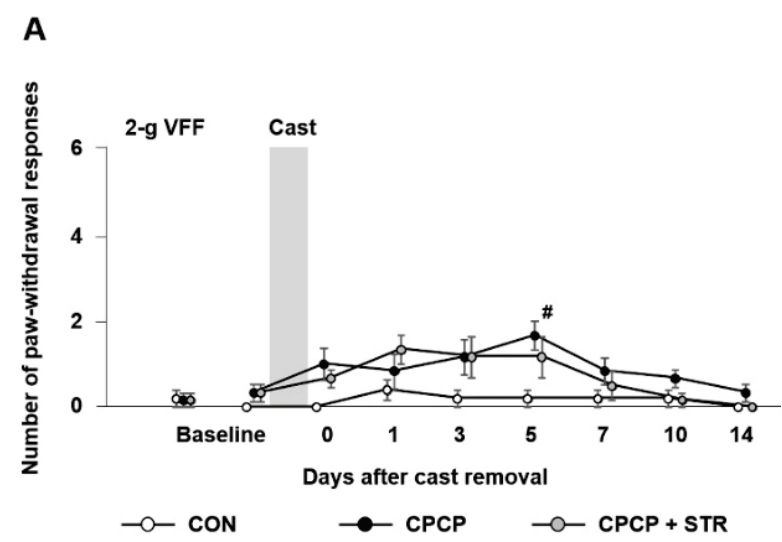

B

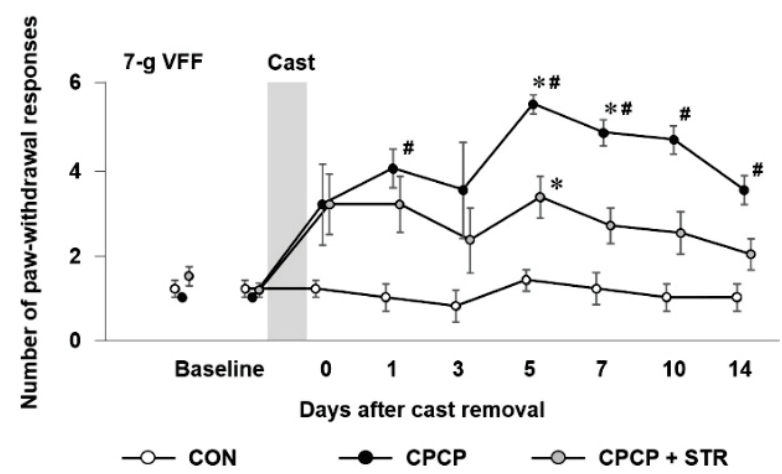

Fig. 3. Time course of changes in number of paw-withdrawal responses. (A) Measurement of mechanical allodynia with 2-g von Frey filament (VFF). (B) Measurement of mechanical hyperalgesia with 7-g VFF. Horizontal axis indicates measurement time points. Data are presented as mean $\pm \operatorname{SEM}(n=5$ or 6$)$. $* P<0.05$ relative to baseline values; ${ }^{\#} P<0.05$ relative to CON group. 
$(P<0.001$ in both hindlimbs in each group). The ROM gradually recovered over the study period. The ROM in the CPCP group was significantly lower than that in the CON group over the 14-day period (right: $P=0.037$ on Day $0, P=0.003$ on Day $1, P=0.004$ on Day $3, P<0.001$ on Days 5, 7, 10, and 14; left: $P=0.018$ on Day $0, P=0.006$ on Day $1, P<0.001$ on Days 3, 5, 7, 10, and 14). Conversely, the $\mathrm{ROM}$ in the $\mathrm{CPCP}+\mathrm{STR}$ group did not significantly differ from than in the CON group on Day 3 or later.
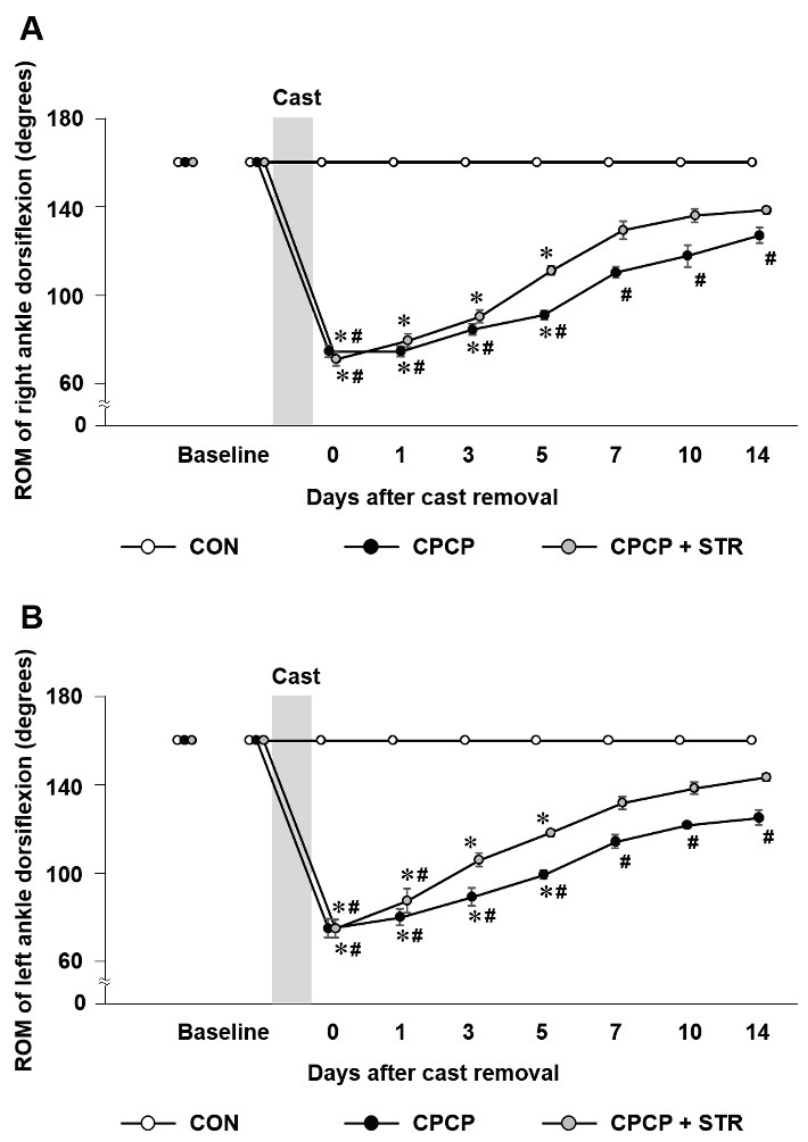

Fig. 4. Time course of changes in range of motion (ROM) of ankle dorsiflexion. (A) ROM of right ankle dorsiflexion. (B) ROM of left ankle dorsiflexion. Horizontal axis indicates measurement time points. Data are presented as mean \pm SEM $(n=5$ or 6$)$. * $P<0.05$ relative to associated baseline values; ${ }^{\#} P<0.05$ relative to CON group.

\section{Histological observations}

The gastrocnemius muscles of age-matched nonimmobilized control rats (CON group) displayed few myofibers with inflammatory infiltration or central nuclei. Conversely, cellular infiltration and central nuclei were evident in the immobilized gastrocnemius muscles (CPCP group) at 14 days following cast removal.

To assess the effects of stretching exercises on the number of fibers with inflammatory infiltration and central nuclei, we evaluated the number of myofibers with these findings per 10,000 fibers in each group. As shown in Figures $5 \mathrm{~A}$ and $\mathrm{B}$, the number of fibers with infiltration and the number with central nuclei were both significantly higher in the CPCP group than in the CON group (both $P=0.004$ ). Conversely, the number of fibers with infiltration and the number with central nuclei in the CPCP+STR group did not significantly differ from numbers in the CON group.

\section{Discussion}

Limb immobilization can cause prolonged joint contracture, muscle injury, and hyperalgesia, which can affect quality of life and increase healthcare costs. The present study revealed that cyclic stretching after hindlimb cast immobilization alleviated hyperalgesia, improved ROM, and limited muscle injury in a rat model of CPCP.

In this study, we used withdrawal responses to evaluate CPCP. Both immobilization groups (CPCP and $\mathrm{CPCP}+\mathrm{STR}$ ) had significantly lower pain thresholds on Day 0 after cast removal than at baseline, which confirms post-immobilization hyperalgesia in our model. However, the group treated with cyclic stretching had rapid amelioration of CPCP, with levels not significantly different from those in the control group by Day 1 after cast removal. Conversely, the CPCP group that was not treated with cyclic stretching had persistently low pain thresholds throughout the 2-week study period. These results are consistent with those of Morimoto et al. (2013), who reported that stretching ameliorated longlasting hyperalgesia, joint limitation, and muscle atrophy induced by cast immobilization in rats. However, our study differed from that of Morimoto in the following respects. First, rats in the present study had a 4-week period of bilateral immobilization from just above the knee to the distal paw, whereas the previous study applied 2 weeks of unilateral immobilization from the trunk to the mid-hindpaw. Second, the present study used cyclic stretching applied six times/week for 2 weeks whereas the previous study used static stretching applied three times/week for 2 weeks. In a preliminary unpublished study, we compared the effects of static versus cyclic stretching on muscle atrophy (fiber crosssectional area) and injury (necrotic fiber number) after immobilization (Supplementary Methods section and Supplementary Tables 1 and 2). We found that cyclic stretching was superior to static stretching in ameliorating 
these conditions. Finally, stretching in the present study was initiated on the day of cast removal, before reloading, whereas stretching was initiated on Day 3 after cast removal in the study of Morimoto et al. (2013). The very early application of passive stretching resulted in significant amelioration in CPCP within 1 day of cast removal in the present study.
A

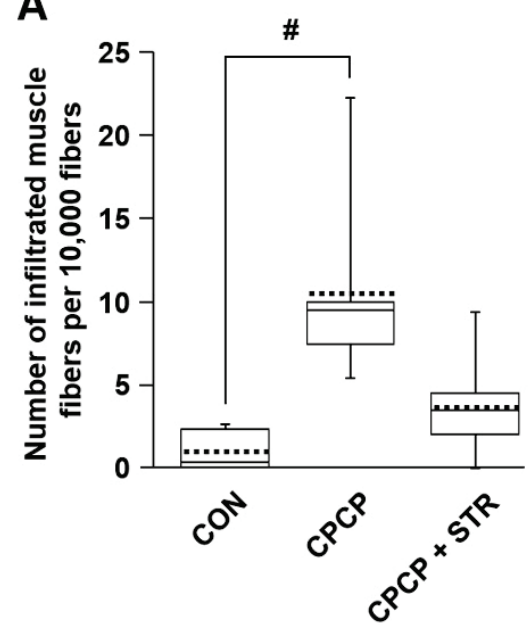

B

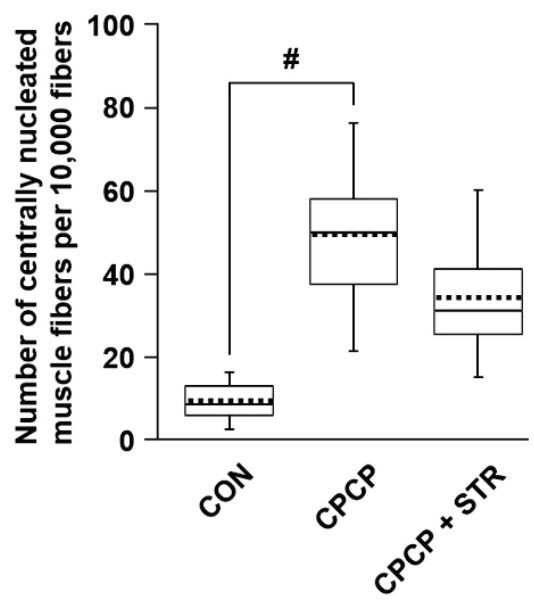

Fig. 5. Effects of stretching exercises on number of muscle fibers with inflammatory infiltration and central nuclei. Histological findings were confirmed with quantitative analysis comparing age-matched naïve controls (CON, $n=5), \mathrm{CPCP}$ rats without cyclic stretching exercise (CPCP, $\mathrm{n}=6)$, and CPCP rats with cyclic stretching exercise (CPCP+STR, $\mathrm{n}=6$ ). (A) Number of infiltrated muscle fibers. (B) Number of centrally nucleated fibers. Values are expressed as box-and-whisker plots (highest, third quartile, median, first quartile, and lowest values). Dotted lines indicate mean values. $\# P<0.05$ relative to CON group.
Joint contracture occurs during immobilization because of structural alterations, including muscle fibrosis and joint capsule changes (Wong et al. 2015). Studies have reported conflicting evidence regarding the efficacy of stretching in the treatment of immobilizationinduced joint contracture. Several studies in animal models have found that stretching significantly improves joint ROM after immobilization (Inoue et al. 2007, Morimoto et al. 2013). However, a recent systematic review of 18 studies found that stretching did not have clinically important effects on joint contracture caused by various etiologies (Harvey et al. 2017). The present results support the efficacy of cyclic stretching in increasing the ROM of joints with immobilizationinduced contracture.

In the present study, we used the presence of central nuclei and inflammatory cells within myofibers as markers of muscle injury. We found higher numbers of infiltrated and centrally nucleated muscle fibers in the gastrocnemius muscles of rats who underwent a 4-week immobilization period than in control rats. Central nuclei are a sign of muscle repair and are seen in various types of muscular dystrophy and after muscle injury (Folker and Baylies 2013). The calf muscles of CPCP rats show disuse atrophy (Inoue et al. 2007); reloading of muscles with disuse atrophy induces inflammatory changes (Frenette et al. 2002). Therefore, the muscle injury in the present study may have resulted from reloading of the atrophic calf muscle. We found that early implementation of cyclic stretching significantly attenuated immobilization-induced muscle injury. This finding is consistent with that of Inoue et al. (2009), who demonstrated that stretching exercises performed soon after cast removal in rats decreased muscle injury (assessed based on inflammatory infiltration and heat shock proteins) in the cast-immobilized hindlimb. Similarly, Gomes et al. (2007) demonstrated that stretching exercises protected rat gastrocnemius muscles from atrophy and muscle damage during disuse. Although the relationship between muscle injury and CPCP is not clear, our finding that stretching decreased muscle injury and alleviated pain suggests that muscle damage may play a role in the development of CPCP. Further studies are needed to clarify this relationship.

This study has several limitations. First, it did not investigate the epidermis, spinal plasticity, or oxidative stress. Second, muscle injury was assessed with two parameters on hematoxylin-eosin staining only. Evaluation of additional histopathologic and systemic parameters could enhance our understanding of the effects of stretching on CPCP. Further detailed investigation of these aspects will be useful to elucidate the mechanisms by which stretching exercises decrease the pain associated with cast immobilization. In addition, Schwann cells and muscle spindles could also be potential targets for exploring the mechanisms.

In conclusion, early implementation of cyclic stretching exercises ameliorated cutaneous and muscular 
mechanical hyperalgesia, joint contracture, and immobilization-induced muscle injury in a rat model of CPCP. Stretching exercises may decrease long-lasting hyperalgesia in patients undergoing rehabilitation following cast immobilization.

\section{Conflict of Interest}

There is no conflict of interest.

\section{Acknowledgements}

The authors are grateful to Yuji Takahashi (Showa University Koto Toyosu Hospital, Japan) for technical assistance in performing the experiments. This work was supported by JSPS KAKENHI Grant Number 22500455 from the Japan Society for the Promotion of Science. We thank Rebecca Tollefson, DVM, from Edanz Group (https://en-author-services.edanzgroup.com/) for editing a draft of this manuscript.

\section{References}

ALLEN G, GALER BS, SCHWARTZ L: Epidemiology of complex regional pain syndrome: a retrospective chart review of 134 patients. Pain 80: 539-544, 1999. https://doi.org/10.1016/s0304-3959(98)00246-2

COREY SM, VIZZARD MA, BOUFFARD NA, BADGER GJ, LANGEVIN HM: Stretching of the back improves gait, mechanical sensitivity and connective tissue inflammation in a rodent model. PLoS One 7: e29831, 2012. https://doi.org/10.1371/journal.pone.0029831

FOLKER E, BAYLIES M: Nuclear positioning in muscle development and disease. Front Physiol 363: 4, 2013. https://doi.org/10.3389/fphys.2013.00363

FRENETTE J, ST-PIERRE M, CÔTÉ CH, MYLONA E, PIZZA FX: Muscle impairment occurs rapidly and precedes inflammatory cell accumulation after mechanical loading. Am J Physiol Regul Integr Comp Physiol 282: R351-R357, 2002. https://doi.org/10.1152/ajpregu.00189.2001

GOMES AR, CORNACHIONE A, SALVINI TF, MATTIELLO-SVERZUT AC: Morphological effects of two protocols of passive stretch over the immobilized rat soleus muscle. J Anat 210: 328-335, 2007. https://doi.org/10.1111/j.1469-7580.2007.00697.x

HAMAUE Y, NAKANO J, SEKINO Y, CHUGANJI S, SAKAMOTO J, YOSHIMURA T, OKITA M, ORIGUCHI T: Effects of vibration therapy on immobilization-induced hypersensitivity in rats. Phys Ther 95: 1015-1026, 2015. https://doi.org/10.2522/ptj.20140137

HARVEY LA, KATALINIC OM, HERBERT RD, MOSELEY AM, LANNIN NA, SCHURR K: Stretch for the treatment and prevention of contracture: an abridged republication of a Cochrane systematic review. J Physiother 63: 67-75, 2017. https://doi.org/10.1016/j.jphys.2017.02.014

HONDA Y, SAKAMOTO J, NAKANO J, KATAOKA H, SASABE R, GOTO K, TANAKA M, ORIGUCHI T, YOSHIMURA T, OKITA M: Upregulation of interleukin- $1 \beta /$ transforming growth factor- $\beta 1$ and hypoxia relate to molecular mechanisms underlying immobilization-induced muscle contracture. Muscle Nerve 52: 419-427, 2015. https://doi.org/10.1002/mus.24558

HONDA Y, TANAKA M, TANAKA N, SASABE R, GOTO K, KATAOKA H, SAKAMOTO J, NAKANO J, OKITA M: Relationship between extensibility and collagen expression in immobilized rat skeletal muscle. Muscle Nerve 57: 672-678, 2018. https://doi.org/10.1002/mus.26011

INOUE T, OKITA M, TAKAHASHI Y, HARADA Y, SUZUKI S: Effect of intermittent stretching on limitation of ankle joint mobility and disuse muscle atrophy in immobilized rat soleus muscle. (In Japanese) Rigaku Ryōhōgaku 34: 1-9, 2007.

INOUE T, SUZUKI S, HAGIWARA R, IWATA M, BANNO Y, OKITA M: Effects of passive stretching on muscle injury and HSP expression during recovery after immobilization in rats. Pathobiology 76: 253-259, 2009. https://doi.org/10.1159/000228901

KANEGUCHI A, OZAWA J, YAMAOKA K: Intra-articular injection of mitomycin C prevents progression of immobilization-induced arthrogenic contracture in the remobilized rat knee. Physiol Res 69: 145-156, 2020. https://doi.org/10.33549/physiolres.934149 
KATAOKA H, NAKANO J, MORIMOTO Y, HONDA Y, SAKAMOTO J, ORIGUCHI T, OKITA M, YOSHIMURA T: Hyperglycemia inhibits recovery from disuse-induced skeletal muscle atrophy in rats. Physiol Res 63: 465-474, 2014.

KOH TJ, PETERSON JM, PIZZA FX, BROOKS SV: Passive stretches protect skeletal muscle of adult and old mice from lengthening contraction-induced injury. J Gerontol A Biol Sci Med Sci 58: 592-597, 2003. https://doi.org/10.1093/gerona/58.7.b592

MAEZAWA T, TANAKA M, KANAZASHI M, MAESHIGE N, KONDO H, ISHIHARA A, FUJINO H: Astaxanthin supplementation attenuates immobilization-induced skeletal muscle fibrosis via suppression of oxidative stress. J Physiol Sci 67: 603-611, 2017. https://doi.org/10.1007/s12576-016-0492-X

MATSUMOTO Y, NAKANO J, OGA S, KATAOKA H, HONDA Y, SAKAMOTO J, OKITA M: The non-thermal effects of pulsed ultrasound irradiation on the development of disuse muscle atrophy in rat gastrocnemius muscle. Ultrasound Med Biol 40: 1578-1586, 2014. https://doi.org/10.1016/j.ultrasmedbio.2013.12.031

MORIMOTO A, WINAGA H, SAKURAI H, OHMICHI M, YOSHIMOTO T, OHMICHI Y, MATSUI T, USHIDA T, OKADA T, SATO J: Treadmill running and static stretching improve long-lasting hyperalgesia, joint limitation, and muscle atrophy induced by cast immobilization in rats. Neurosci Lett 534: 295-300, 2013. https://doi.org/10.1016/j.neulet.2012.11.009

NAKABAYASHI K, SAKAMOTO J, KATAOKA H, KONDO Y, HAMAUE Y, HONDA Y, NAKANO J, OKITA M: Effect of continuous passive motion initiated after the onset of arthritis on inflammation and secondary hyperalgesia in rats. Physiol Res 65: 683-691, 2016. https://doi.org/10.33549/physiolres.933214

NAKAGAWA T, HIRAGA SI, MIZUMURA K, HORI K, OZAKI N, KOEDA T: Topical thermal therapy with hot packs suppresses physical inactivity-induced mechanical hyperalgesia and up-regulation of NGF. J Physiol Sci 68: 629-637, 2018. https://doi.org/10.1007/s12576-017-0574-4

NAKANO J, SEKINO Y, HAMAUE Y, SAKAMOTO J, YOSHIMURA T, ORIGUCHI T, OKITA M: Changes in hind paw epidermal thickness, peripheral nerve distribution and mechanical sensitivity after immobilization in rats. Physiol Res 61: 643-647, 2012. https://doi.org/10.33549/physiolres.932362

NASU T, TAGUCHI T, MIZUMURA K: Persistent deep mechanical hyperalgesia induced by repeated cold stress in rats. Eur J Pain 14: 236-244, 2010. https://doi.org/10.1016/j.ejpain.2009.05.009

OHMICHI Y, SATO J, OHMICHI M, SAKURAI H, YOSHIMOTO T, MORIMOTO A, HASHIMOTO T, EGUCHI K, NISHIHARA M, ARAI YC, OHISHI H, ASAMOTO K, USHIDA T, NAKANO T, KUMAZAWA T: Two-week cast immobilization induced chronic widespread hyperalgesia in rats. Eur J Pain 16: 338-348, 2012. https://doi.org/10.1002/j.1532-2149.2011.00026.x

OKITA M, NAKANO J, KATAOKA H, SAKAMOTO J, ORIGUCHI T, YOSHIMURA T: Effects of therapeutic ultrasound on joint mobility and collagen fibril arrangement in the endomysium of immobilized rat soleus muscle. Ultrasound Med Biol 35: 237-244, 2009. https://doi.org/10.1016/j.ultrasmedbio.2008.09.001

PELESHOK JC, RIBEIRO-DA-SILVA A: Delayed reinnervation by nonpeptidergic nociceptive afferents of the glabrous skin of the rat hindpaw in a neuropathic pain model. J Comp Neurol 519: 49-63, 2011. https://doi.org/10.1002/cne.22500

SEKINO Y, NAKANO J, HAMAUE Y, CHUGANJI S, SAKAMOTO J, YOSHIMURA T, ORIGUCHI T, OKITA M: Sensory hyperinnervation and increase in NGF, TRPV1 and P2X3 expression in the epidermis following cast immobilization in rats. Eur J Pain 18: 639-648, 2014. https://doi.org/10.1002/j.1532-2149.2013.00412.x

TERKELSEN AJ, BACH FW, JENSEN TS: Experimental forearm immobilization in humans induces cold and mechanical hyperalgesia. Anesthesiology 109: 297-307, 2008. https://doi.org/10.1097/aln.0b013e31817f4c9d

YOSHIMURA A, SAKAMOTO J, HONDA Y, KATAOKA H, NAKANO J, OKITA M: Cyclic muscle twitch contraction inhibits immobilization-induced muscle contracture and fibrosis in rats. Connect Tissue Res 58: 487-495, 2017. https://doi.org/10.1080/03008207.2016.1257004

WONG K, TRUDEL G, LANEUVILLE O: Noninflammatory joint contractures arising from immobility: animal models to future treatments. Biomed Res Int 2015: 848290, 2015. https://doi.org/10.1155/2015/848290 
ZSCHÜNTZSCH J, ZHANG Y, KLINKER F, MAKOSCH G, KLINGE L, MALZAHN D, BRINKMEIER H, LIEBETANZ D, SCHMIDT J: Treatment with human immunoglobulin $\mathrm{G}$ improves the early disease course in a mouse model of Duchenne muscular dystrophy. J Neurochem 136: 351-362, 2016. https://doi.org/10.1111/jnc.13269 